\title{
Can psychosocial and socio-demographic questions help identify sexual risk among heterosexually-active women of reproductive age? Evidence from Britain's third National Survey of Sexual Attitudes and Lifestyles (Natsal-3)
}

Natalie Edelman ${ }^{1,2}$, Jackie A. Cassell ${ }^{1}$, Richard de Visser ${ }^{3}$, Philip Prah ${ }^{4}$ and Catherine H. Mercer ${ }^{4^{*}}$

\begin{abstract}
Background: Contraceptive advice and supply (CAS) and sexually transmitted infection (STI) testing are increasingly provided in primary care. Most risk assessment tools are based on sexual risk behaviours and socio-demographics, for use online or in specialist services. Combining socio-demographic and psychosocial questions (e.g. religious belief and formative experience) may generate an acceptable tool for targeting women in primary care who would benefit from intervention. We aimed to identify psychosocial and socio-demographic factors associated with reporting key sexual risk behaviours among women in the British general population.

Methods: We undertook complex survey analysis of data from 4911 hetero-sexually active women aged 16-44 years, who participated in Britain's third National Survey of Sexual Attitudes and Lifestyles (Natsal-3), a national probability sample survey undertaken 2010-2012. We used multivariable regression to examine associations between the available psychosocial and socio-demographic variables in Natsal-3 and reports of three key sexual behaviours: a) 2+ partners in the last year (2PP); b) non-use of condoms with $2+$ partners in the last year (2PPNC); c) non-use of condoms at first sex with most recent sexual partner (FSNC). We adjusted for key socio-demographic factors: age, ethnicity and socioeconomic status (measured by housing tenure).

Results: Weekly binge drinking (6+ units on one occasion), and first sex before age 16 were each positively associated with all three sexual behaviours after adjustment. Current relationship status, reporting drug use (ever), younger age and living in rented accommodation were also associated with $2+$ partners and $2+$ partners without condoms after adjustment. Currently being a smoker, older age and respondent ethnicity were associated with FSNC after adjustment for all other variables. Current smoking status, treatment for depression (last year), and living at home with both parents until the age of 14 were each associated with one or more of the behaviours.

Conclusions: Reported weekly binge drinking, early sexual debut, and age group may help target STI testing and/or CAS among women. Further research is needed to examine the proportion of sexual risk explained by these factors, the acceptability of these questions to women in primary care and the need to customise them for community and other settings.
\end{abstract}

Keywords: Sexual risk, Survey, Psychosocial, Socio-demographic, Women

\footnotetext{
* Correspondence: c.mercer@ucl.ac.uk

${ }^{4}$ Centre for Sexual Health and HIV Research, University College London, 3rd

Floor Mortimer Market Centre off Capper Street, London WC1E 6JB, UK

Full list of author information is available at the end of the article
} 


\section{Background}

Much sexual health research to date has focused on how sexual behaviour is associated with acquisition and transmission of sexually transmitted infection (STI), and to a lesser degree- with unplanned pregnancy (UP) [1]. These approaches have supported the identification of higher risk populations (such as young people and Men who have Sex with Men), and the targeting of these populations with sexual health interventions, e.g. England's National Chlamydia Screening Programme [2]. Accordingly, a number of sexual risk assessment tools have been developed for clinical use. Although none of these address risk of UP, several have been developed to identify those at risk of STIs, typically based on sexual behaviour and socio-demographic items such as age, ethnicity, gender and sexual orientation $[3,4]$.

There is also growing evidence that 'psychosocial' factors such as relationship qualities [5, 6], mental health [7], and substance use [8] may also be associated with adverse sexual health outcomes, and with the sexual risk behaviours which mediate them. Thus, they may be of use in identifying at-risk individuals for targeted intervention. Within social epidemiology, psychosocial factors have been defined as those which influence health outcomes by affecting biology, behaviour or psychology [9]. For the purposes of this study we define it as factors which do not fit within the categories of socio-demographics, sexual behaviour or health psychology constructs such as risk perception and self-efficacy. Previous use of this definition identified factors that concern health, substance use, formative experiences, lifestyle, and relationships [10].

This exploratory study was undertaken to underpin the development of a psychosocial clinical prediction rule (CPR) [11], being developed primarily for use in targeting STI testing, safe sex advice and contraception advice and supply (CAS) to women of reproductive age attending British primary care settings. As such, this study reflects a biomedical understanding of women's sexual health risk, contrasting with the broader definition of sexual health endorsed by the World Health Organization [12]. CPRs are tools used in clinical assessment to direct the nature of clinical intervention, based on response to a short set of patient questions. The CPR which this work underpins is envisaged as a brief questionnaire which women can selfcomplete in order to assess their need for STI testing and/ or CAS. In this exploratory study we hypothesised that combining psychosocial and socio-demographic factors may help explain variance in sexual risk behaviour within populations, enabling targeting of those at greater risk of adverse sexual health outcomes without the need for clinical staff to take a sexual history. This may be advantageous as sexual history-taking can be time-consuming for staff and patient; and may be perceived as intrusive where the patient has attended primary care for non-sexual health matters. This approach may also flag up psychosocial issues, such as binge-drinking, which warrant treatment in their own right and may also be antecedents of a broader range of sexual risk - such as risk of STIs through partners, and inconsistent contraception use.

Therefore our aim was to explore the extent to which psychosocial and socio-demographic factors might be used to identify women experiencing higher levels of sexual risk. As a proxy, in this study we examined hypothesised associations between psychosocial and socio-demographic factors with key sexual risk behaviours, using data from a national probability sample survey.

To this end we address three research questions:

1. Which psychosocial and socio-demographic factors are associated with key sexual risk behaviours?

2. Which psychosocial and socio-demographic factors, if any, are associated across different sexual risk behaviours?

3. Do observed associations between psychosocial factors and sexual risk behaviours remain after adjustment for key socio-demographic factors: age group, ethnicity and socio-economic status (measured by housing tenure)?

\section{Methods}

Data from the third National Survey of Sexual Attitudes and Lifestyles (Natsal-3) were analysed. This probability sample survey of the British resident population aged 16-74 years was conducted from 2010 to 2012 inclusive, using a multi-stage, clustered and stratified sampling methodology. In line with standard practice for UK surveys, and in response to evidence suggesting that signing a consent form might lead to a greater sense of obligation to complete the interview, we obtained verbal rather than written consent. Full details of the study design are described elsewhere [13, 14]. A sample size of 15,162 was achieved - an overall response rate of $57.7 \%$ with only very small (typically 1-3\%) amounts of item nonresponse [13]. Natsal-3 asked about subjects, including sexual partners and practices, experience of depression, substance use, STI diagnosis, unplanned pregnancy, sexual function [15] and non-volitional sex [16]. This has permitted investigation of the wider social and health contexts of sexual health and behaviour [17].

The Natsal-3 dataset provides an opportunity to examine the existence of associations in the general population; which, being heterogeneous in sexual risk is broadly representative of a primary care clinic population. This contrasts with specialist sexual health clinic populations which tend to report greater sexual risk behaviour [18]. The analyses presented in this paper were conducted on a subset of Natsal-3 respondents, defined 
as women aged 16-44 years who reported heterosexual sex (defined as anal, vaginal, or oral sex with a male partner) in the last year. These criteria were used to generate findings most applicable to the development of the CPR.

\section{Outcome measures}

Low population prevalence of STI diagnosis, abortion and unplanned pregnancy in the last year, found in this Natsal-3 sample, meant that there was insufficient statistical power to analyse these as outcomes in this study. Instead three sexual risk behaviour measures [19] were selected as proxies for these adverse sexual health outcomes, on the grounds that STI screening and CAS may be offered in response to reported sexual risk behaviour.

To minimise the risk of Type II error in the analysis we selected three key sexual risk behaviour variables which were reported by more than $10 \%$ of the subpopulation of interest, equating to $n \geq 500$ women. Having $2+$ partners in the last year (abbreviated here as $2 \mathrm{PP}$ ) was chosen as a variable known to be associated with STI acquisition and commonly used for clinical and research purposes $[20,21]$. Non-use of condoms with 2+ partners in the last year (abbreviated here as 2PPNC) was chosen as it combines multiple partnerships and condom use to provide a more precise indicator of STI risk behaviour. Specifically this variable refers to at least one episode of non-use of condoms, occurring with two or more partners. Finally, we selected non-use of condoms at first sex with most recent partner (including those only reporting having sex once with their most recent partner), abbreviated here as FSNC. This variable represents a sexual encounter when 'risk' of infection or unplanned pregnancy might be most highly perceived and we might therefore anticipate a greater likelihood of condom use. However, as a single sexual encounter is unlikely to result in significant risk, this exploratory variable constitutes a potential proxy indicator of broader sexual risk experiences. By focusing only on non-use of condoms this variable may also be more representative of those at risk of STIs through partner, rather than own, sexual risk behaviours.

\section{Psychosocial and socio-demographic variables}

As this study was undertaken to support the development of a CPR, investigation focused on identifying socio-demographic and psychosocial items strongly and commonly associated with measures of sexual risk, which would also be brief, acceptable and easy to score as clinical questionnaire items.

Most of the psychosocial variables selected for testing in bivariate analysis fitted into one of the following broad topics: substance use, mental health, general health, sexual orientation, formative experiences involving family, formative experiences involving sex, partner descriptors and relationship status and satisfaction. We chose a measure of sexual orientation that did not incorporate sexual behaviour. This was driven by a decision to avoid incorporating sexual behaviour items as exposures, coupled with a concern that sexual orientation items which are defined by sexual behaviour with men and women, may be confounded by multiple partnerships.

Questions asked in Natsal-3 regarding non-volitional sex [16] were deemed unlikely to be acceptable for use in a CPR and were therefore excluded from our analyses. Childhood sexual abuse (CSA) was however included covertly, within a dichotomous variable constructed for first heterosexual intercourse $<16$ years (yes/no), as there is evidence of correlations between sexual risk and morbidity, CSA and early sexual debut [22]. This contrasts with other Natsal-3 papers which report on first heterosexual intercourse $<16$ years, but exclude from their analysis those reporting first sex under the age of 13 years [23, 24].

Where more than one brief and easy-to-score psychosocial variable remained within the same topic and each was found to be associated with the outcomes of interest, the variable with the highest frequency of response was selected for multivariable analysis. This approach was founded on the rationale that rarer psychosocial factors will explain a low proportion of variance in sexual risk behaviour (no matter how strong the association) and will therefore have less utility in the general population. This corresponds with the notion of 'adequate prediction' [11].

Forty-two variables were initially identified from the Natsal-3 dataset as being representative of psychosocial factors (see Additional file 1). The original questions from which they were derived can be viewed at http://www.natsal.ac.uk/natsal-3/core-survey/ questionnaire.aspx. Of the 42 variables identified, ten were selected by applying the approach described above. These were:

- relationship status (recoded as cohabiting with partner, stable relationship not cohabiting, not in a relationship but previously cohabited, not in a relationship and never cohabited)

- sexual identity (recoded as heterosexual/not heterosexual)

- belong to any religion now (coded as yes/no)

- smokes cigarettes nowadays (recoded as yes/no)

- weekly drinking of $6+$ units of alcohol on one occasion (recoded as yes/no)

- ever taken non-prescribed drugs (yes/no)

- received treatment for depression in the last year (recoded as yes/no)

- lived with both parents until the age of 14 (yes/no) 
- first heterosexual intercourse before the age of 16 years (yes/no)

- most recent partner's ethnicity (white/Asian British/ black British/other)

There is strong existing evidence for associations between sexual morbidity and socio-demographic factors $[25,26]$. Therefore age group [15], ethnicity and socioeconomic status were also assessed for model inclusion, using rental of current home as a brief and acceptable proxy indicator of the latter.

\section{Statistical methods}

In order to make the sample broadly representative of the target population according to the 2011 Census, selection probability weights were applied to the data to adjust for the unequal probability of selection and then post-stratification weights were applied to adjust for non-response bias [14].

Aggregated variable categories were used only where it was necessary to boost cell frequencies or render items briefer and easier to complete. Aggregation was based only on observed gaps in continuous data or on overlapping confidence intervals for categorical data - with the exception of body weight which was aggregated using the weight boundaries for body mass index (BMI) on a typical 5'7" woman. This approach reduced the number of bivariate analyses and therefore of Type 1 error. Hence, we did not apply a Bonferroni correction to our analyses [27].

Bivariate analyses were conducted with each psychosocial and socio-demographic variable described above, for each of the three sexual risk behaviour variables. Those which demonstrated an association at $p \leq 0.05$ were entered into a multivariable model for that behaviour. Psychosocial variable associations between $0.05 \leq p<0.10$ (after adjustment for socio-demographic variables) were also entered. This approach provided consistency with criteria for removal of variables from the model which was set at $p \geq 0.1$. Backwards stepwise multivariable logistic regression was used for the chosen psychosocial and socio-demographic variables to identify which combination of these were retained in the model using pre-set criteria. This approach was chosen as many concerns with this type of analysis were reduced in this study. I.e. we selected empirically only a modest number of variables, and risk of Type II error was reduced by the large sample size and by setting the criteria for removal of the model at $p \geq 0.1$. This criterion, and the backwards elimination approach, also acted to reduce suppressor effects [37]. Table 1 in the Results section presents the percentage reporting that risk behaviour in each category of each exposure, the crude odds ratios for each category of that exposure, and the global $\mathrm{P}$ value for each exposure. For each of the exposures entered into the multivariable model, Table 1 also presents the global $\mathrm{P}$ value, with adjusted odds ratios for each exposure that was retained after adjustment for all other variables.

\section{Results}

This analysis included 4911 women aged 16-44 years who reported having heterosexual sex in the year prior to their Natsal-3 interview. Sample characteristics are given in Table 1 which also presents the model for each of the sexual behaviours analysed.

Having 2+ partners in the last year (2PP) was reported by $17.9 \%$ of respondents. Non-use of condoms with $2+$ partners in the last year (2PPNC) was reported by $9.8 \%$ of respondents. Non-use of condoms at first sex with most recent partner (including those only have sex once with their most recent partner (FSNC) was reported by $41.5 \%$ of respondents. FSNC showed only small overlap with the other two behaviours - among these $41.5 \%$ of respondents, $8.5 \%$ reported $2 \mathrm{PP}$ in the last year and $6.3 \%$ reported 2PPNC in the last year.

First heterosexual intercourse at age $<16$ years and weekly binge drinking in the last year were each associated with all three sexual risk behaviours after adjustment for both socio-demographic variables and all other psychosocial variables, with adjusted odds ratios (AOR) ranging from 1.16 to 14.16 . In contrast, two psychosocial variables - sexual identity and most recent sexual partner's ethnicity - were not associated with any of the three sexual behaviours in multivariable regression.

Of the socio-demographic factors analysed, not owning a property was associated with both $2 \mathrm{PP}$ and $2 \mathrm{PPNC}$ $(p<0.05)$ after adjustment for all other variables, but only at $(p=0.077)$ with FSNC. Younger age group was positively associated with both $2 \mathrm{PP}$ and 2PPNC (adjusted), although 2PPNC showed no association between those aged 16-24 versus those aged 25-34 years. In contrast, younger women were less likely to report FSNC. After adjustment respondent ethnicity was associated only with FSNC.

Although current smoking and currently belonging to a religion were retained in the model for 2PP they were not retained in the model for 2PPNC. Conversely, treatment for depression in the last year was retained in the model for 2PPNC, but not in the model for 2PP. Not cohabiting with a partner was retained in the models for both 2PP and 2PPNC, but not the model for FSNC. In contrast, the variable for not living with both parents until age 14 was retained in the model for FSNC but not in the models for 2PP or 2PPNC. Although demonstrating a small effect size this exposure was reported by $26.8 \%$ of respondents. 


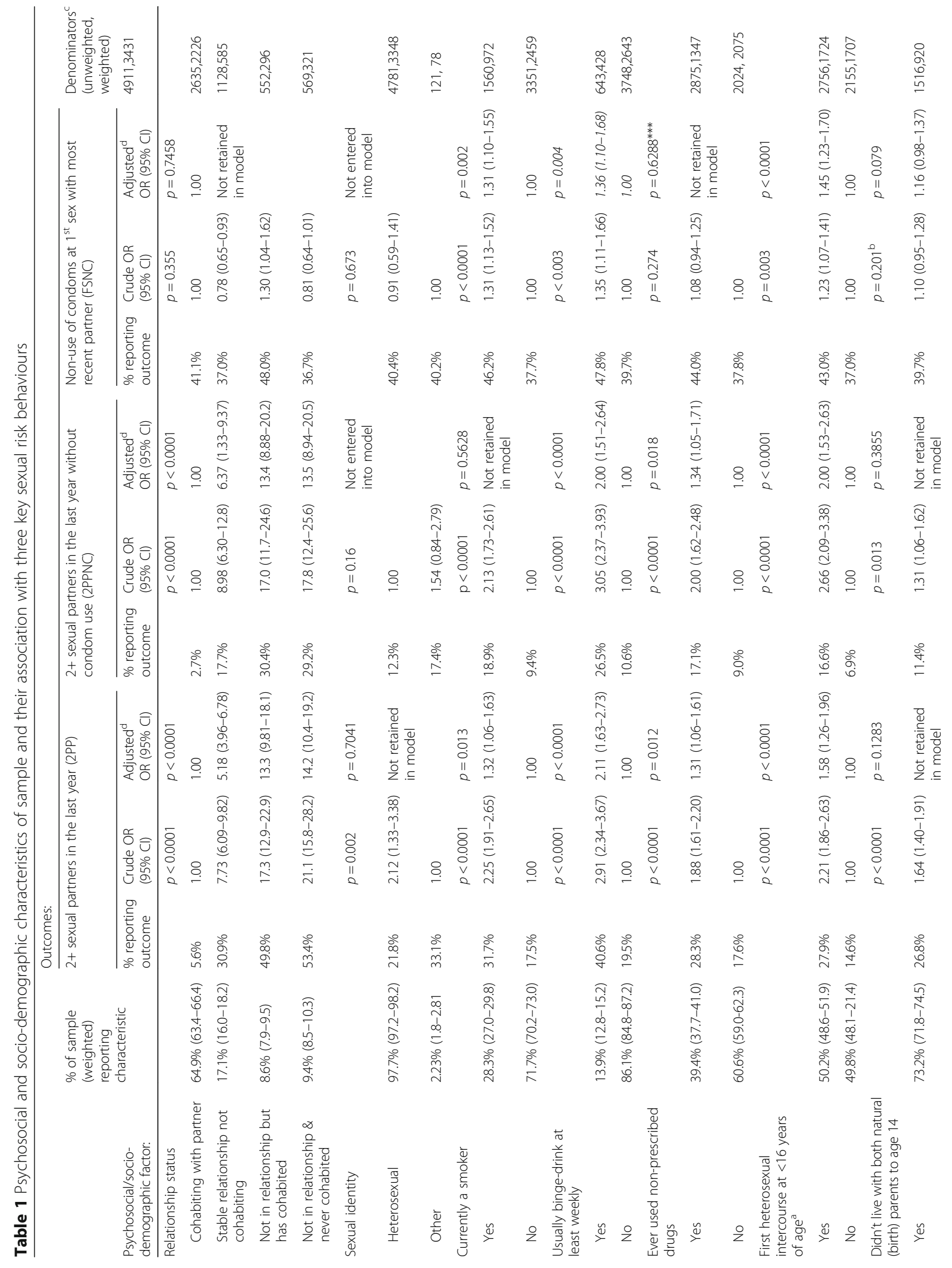




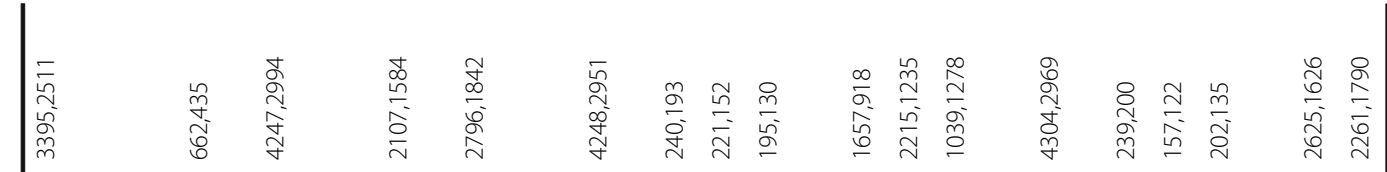

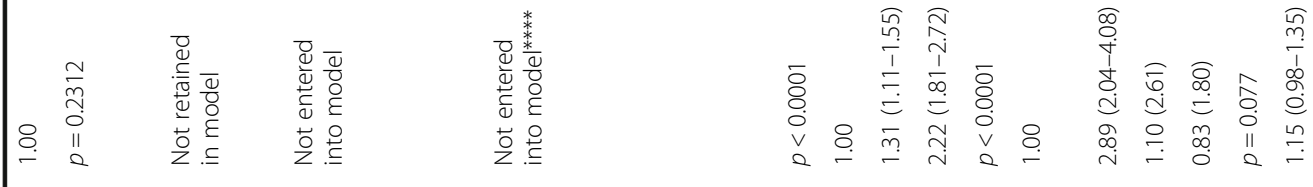

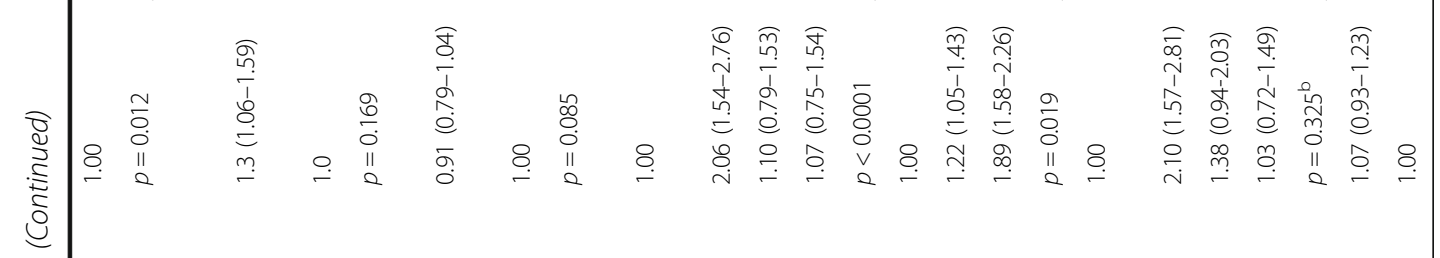

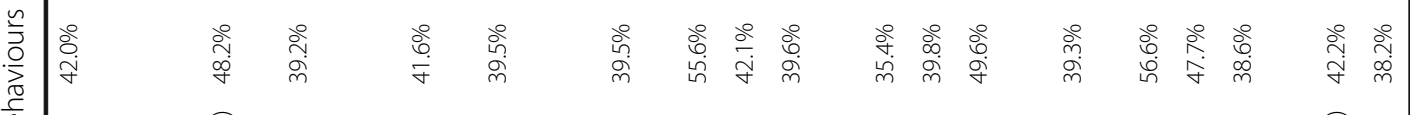

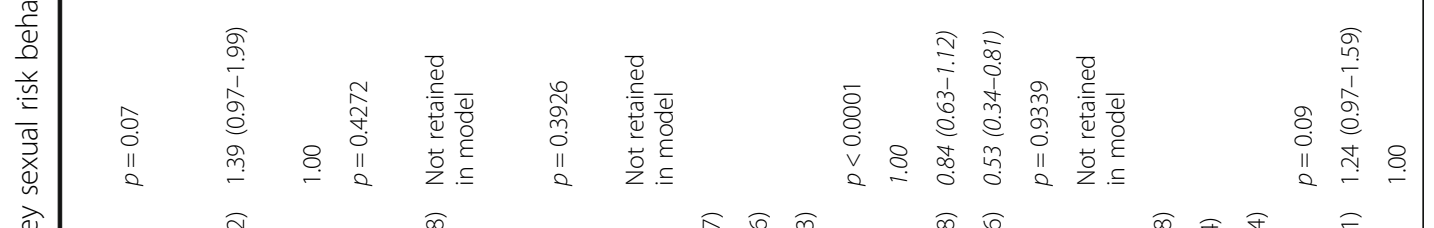

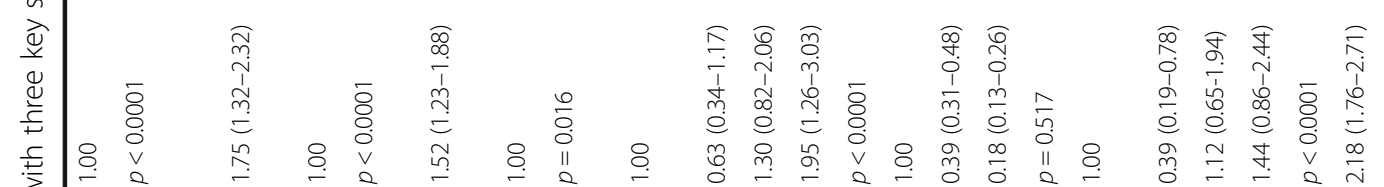

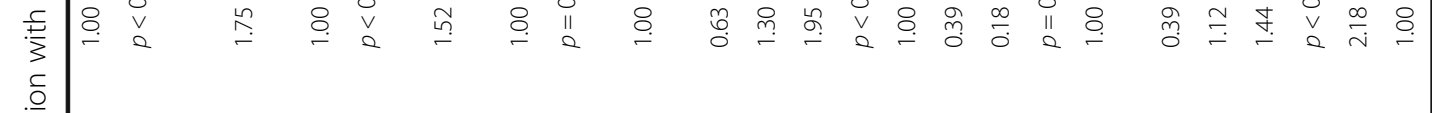

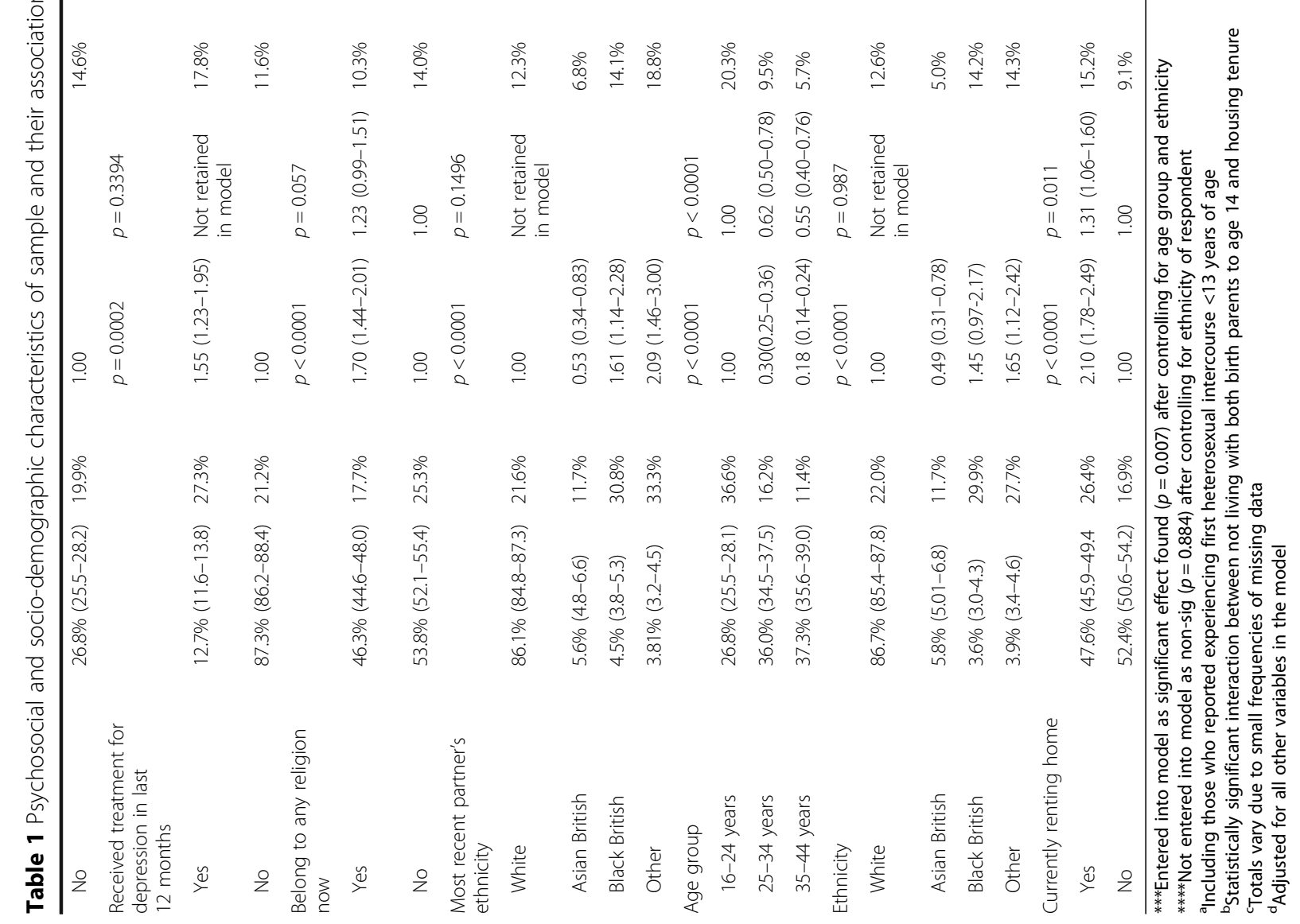


Compared to currently cohabiting with a partner, each of the non-cohabiting response options showed very large effect sizes (odds ratios) in the 2PP and 2PPNC models, alongside high prevalence - not cohabiting but in a stable relationship (17\%) and not being in a stable relationship (cumulatively 18\%). For the $2 \mathrm{PP}$ and $2 \mathrm{PPNC}$ models there was little overlap between 'stable relationship not cohabiting' and the two 'not in a relationship' response options but great overlap between the latter two options, which also showed the greatest magnitude of effect. In the 2PP and 2PPNC models, drug use ever and currently renting showed very modest, though significant, effect sizes. In the model for FSNC largest effect sizes were observed for Black and Asian ethnicity, and for older age group (25-34 years versus 16-24 years, and $35-44$ years versus $16-24$ years).

\section{Discussion}

Reporting weekly binge drinking in the last year, early sexual debut, younger age group and living in rented accommodation showed association with all three of the sexual risk behaviours studied (2PP, 2PPNC and FSNC). Notably, younger age was positively associated with multiple partnerships but negatively associated with FSNC. FSNC also showed quite different patterns of association to 2PP and 2PPNC overall, with much smaller effect sizes. Not living with both parents to the age of 14 years was associated with FSNC after adjustment for other factors, but not with $2 \mathrm{PP}$ or $2 \mathrm{PPNC}$ after adjustment. Not cohabiting with a partner was associated with 2PP and with 2PPNC after adjustment but not with FSNC. This may reflect that FSNC is not a good proxy for recent sexual risk, particularly as this variable was not limited to episodes of first sex occurring within the last year.

Binge drinking, early sexual debut and younger age have also been found to correlate with sexual risk in other population studies of sexual risk among women [10, 23, 28, 29]. However, observed associations between the dichotomous housing tenure variable and sexual risk contrast with previous Natsal-3 analyses which used comprehensive socio-economic variables [13]. Sexual identity was not found to be associated with sexual risk in our analysis. This may reflect one or more of the following: insufficient power to detect a significant association, a focus on heterosexual risk behaviour in defining the population of interest, or use of a sexual orientation measure based on identity rather than behaviour. However, on this latter point, two population surveys using non-behavioural measures of sexual identity have shown differences in partner numbers among adolescent women [30, 31].

Although a number of studies have examined associations between specific psychosocial factors and sexual risk behaviour, this study is unique in examining three sexual risk behaviours using a combination of psychosocial and socio-demographic items, in order to develop a CPR. Our findings are unique in demonstrating that binge drinking, early sexual debut, younger age and housing tenure remain significant when represented by briefly-worded and common variables, and might in principle be used to target sexual health interventions in primary care settings. This contrasts with existing sexual risk tools in two ways. Firstly, none of the existing tools have been developed for use using a population survey dataset. This reflects that only one has been developed for use in primary care (specifically for identifying STIs among paediatric primary care attenders in the United States [32]). Secondly existing tools focus primarily on sexual behaviour, symptoms and socio-demographics [33-35]. Where psychosocial items have been included in other tools, selection was not empirically-based but reflected service intentions to identify and address adjunct issues such as intimate partner violence [36].

Our findings also suggest that different items may indicate different sexual risk experiences. E.g. results indicate that 'not cohabiting with a partner' is likely to perform better than 'drug use ever' in identifying women experiencing multiple partnerships, while specific identification of FSNC would rest on being older and not living with both parents until the age of 14 years. Finally, the symmetrical treatment of both psychosocial and socio-demographic variables in our analysis allowed us to examine associations between sexual risk and sociodemographic factors (namely age group and socioeconomic status) while controlling for psychosocial factors. The findings indicate that these associations are not fully explained by psychosocial factors, and that socio-demographic questions should be combined with psychosocial questions in the CPR under development.

\section{Limitations}

This analysis was limited by the variables available in the Natsal-3 dataset and by the prevalence of some of those variables given the size of the Natsal-3 sample. Hence non-significant findings may reflect a lack of statistical power. Similarly, low prevalence of unplanned pregnancy and of STI diagnoses in the population of interest precluded analysis of these outcomes. As the analysis focuses on women heterosexually active in the previous year, so our findings may not apply to women who were not sexually active in the last year, or who had sex exclusively with women (WSEW) in the last year. Nonetheless a large proportion of WSEW also have sex with men [38].

Unsurprisingly (as 2PPNC is a sub-category of 2PP) greater similarities were seen in the associations for these two overlapping variables. In contrast to $2 \mathrm{PP}$ and 2PPNC, FSNC represents only a proxy measure of risk 
behaviour. I.e., as it concerns only one episode of intercourse which may be non-recent so it cannot be considered a key risk factor for STI acquisition or UP in its own right. It is also important to note that, for some respondents, non-use of condoms may represent attempts to conceive, which is nonetheless considered a sexual risk behaviour for STIs. Natsal-3 was not designed to enable assessment of prospective unplanned pregnancy risk (i.e. lack of effective contraceptive use in those not wishing to become pregnant) and consequently this limited our analysis.

Education is commonly investigated as a proxy measure of socio-economic status. However it may carry psychosocial dimensions with regard to factors such as belonging, purpose, and social cohesion. However, none of the available education variables were suitable for use across all age groups. This reflects a common problem in such studies- that age is a major confounder of both duration of education and qualification attainment. Variables concerning non-volitional sex were also excluded from the analysis, as we anticipated that these questions would be unacceptable in a Primary Care- based assessment, so that measuring and adjusting for them in this analysis was of no practical benefit. This pragmatic approach to adjustment reflects the overarching purpose of this study in developing a CPR for primary care use. This stands in contrast to a conventional complex survey methodology approach - in which all factors likely to confound associations are adjusted for, to achieve a more accurate picture of how an exposure and outcome are independently associated.

Although a conservative approach was undertaken towards aggregation of response categories, wide confidence intervals may have led to aggregation of distinct categories with loss of data sensitivity as a result. Nonetheless, most of the substance use variables were associated with sexual risk outcomes, while very few of the relationship quality variables were; this topic-based pattern suggests that aggregation-based insensitivity was not likely to be responsible for the overall patterns of association.

\section{Conclusions}

This study indicates that there are a number of variables which are worthy of further investigation for use in a Clinical Prediction Rule to identify women experiencing sexual risk in primary care settings, and are suitable for self-completion. Certain socio-demographic and psychosocial variables which were associated with only one or two of the risk behaviours studied may also be useful in differentiating between those needing STI testing or CAS.

From this analysis we cannot draw conclusions about causation. Our working definition of 'psychosocial' may have incorporated variables whose association with sexual risk behaviours represent spurious associations rather than being 'the causes of the causes' [39]. Nonetheless, factors such as binge drinking may constitute wider determinants of sexual health, prevention of which may reduce sexual (and other) morbidity. This is highlighted by England's Sexual Health Improvement Framework, 2013 [40].

We also cannot assume that the performance of questions investigated in Natsal-3 will directly transfer to a clinical prediction rule administered in Primary Care. This is because of differences in purpose (research versus clinical practice) and delivery (random sampling versus clinical delivery). Further research is focusing on the performance of psychosocial and socio-demographic variables as CPR questions in clinical settings - using the variables found in this analysis and in systematic review of relevant literature [10]. This ongoing work by the authors is investigating the degree and type of sexual risk explained by these variables, and their acceptability and delivery as questions used in primary care assessment.

\section{Additional file}

Additional file 1: Description of data: List of Natsal-3 variables initially considered for variable inclusion. (DOCX $20 \mathrm{~kb}$ )

\section{Abbreviations \\ 2PP: 2 or more sexual partners in the last year; 2PPNC: 2 or more sexual partners in the last year with whom a condom was not used; AOR: Adjusted odds ratio; BMl: Body mass index; CAS: Contraceptive advice and supply; CPR: Clinical prediction rule; CSA: Childhood sexual abuse; FSNC: Non-use of condoms at first sex with most recent partner; Natsal-3: National Survey of Sexual Attitudes and Lifestyles; STI: Sexually transmitted infection; UP: Unplanned pregnancy}

\section{Acknowledgements}

The authors would like to acknowledge the Natsal-3 team for allowing access to the dataset, and supporting this analysis.

\section{Funding}

This is a summary of independent research funded by the National Institute for Health Research (NIHR)'s Doctoral Research Fellowship programme. The views expressed are those of the authors and not necessarily those of the NHS, the NIHR or the Department of Health. Natsal-3 is a collaboration between University College London (London, UK), the London School of Hygiene and Tropical Medicine (London, UK), NatCen Social Research, Public Health England (formerly the Health Protection Agency), and the University of Manchester (Manchester, UK). The study was supported by grants from the Medical Research Council (G0701757) and the Wellcome Trust (084840), with contributions from the Economic and Social Research Council and the Department of Health. The funders had no involvement in the study design, data collection, analysis and interpretation of the data, the writing of this manuscript or the decision to submit for publication.

\section{Availability of data and materials}

The dataset supporting the conclusions of this article is available in the UK Data Service repository, unique persistent identifier: 10.5255/UKDA-SN-7799-1; https:/discover.ukdataservice.ac.uk/catalogue/?sn=7799\&type=Data\%20 catalogue. 


\section{Authors' contributions}

NE prepared the data, conducted the analysis, and took the primary role in writing the paper. CM oversaw data preparation and analysis, and advised on paper drafts. PP advised on and checked analyses, RdV and JC advised on paper drafts and the overall approach to analysis. All authors read and approved the final manuscript.

\section{Competing interests}

The authors declare that they have no competing interests.

\section{Consent for publication}

Not applicable.

\section{Ethics approval and consent to participate}

Approval was granted by the Oxfordshire Research Ethics Committee A (Ref: 10/H0604/27) and informed consent obtained for all participants in this study.

\section{Author details}

Brighton \& Sussex Medical School, 318b Mayfield House, Village Way, Brighton, Falmer BN1 9PH, UK. ${ }^{2}$ University of Brighton, Brighton, UK. ${ }^{3}$ School of Psychology, Room 1C12 Pevensey1, University of Sussex, Brighton BN1 9RH, UK. ${ }^{4}$ Centre for Sexual Health and HIV Research, University College London, 3rd Floor Mortimer Market Centre off Capper Street, London WC1E 6JB, UK.

\section{Received: 20 May 2016 Accepted: 8 December 2016} Published online: 04 January 2017

\section{References}

1. Wellings $\mathrm{K}$, Johnson AM. Framing sexual health research: adopting a broader perspective. Lancet. 2013;382(9907):1759-62

2. Johnson SA, Simms I, Sheringham J. The implementation of chlamydia screening: a cross-sectional study in the South East of England. Sex Transm Infect. 2010:86:217-21.

3. Falasinnu T, Gustafson P, Hottes TS, Gilbert M, Ogilvie G, Shoveller J. A critical appraisal of risk models for predicting sexually transmitted infections. Sex Transm Dis. 2014;41(5):321-30.

4. Gaydos CA, Jett-Goheen M, Barnes M, Dize L, Barnes P, Hsieh Y-H. Use of a risk quiz to predict infection for sexually transmitted infections: a retrospective analysis of acceptability and positivity. Sex Transm Infect. 2015;92(1): 44-8.

5. Mittal M, Senn TE, Carey MP. Intimate partner violence and condom use among women: does the information-motivation-behaviour skills mode explain sexual risk behaviour? AIDS Behav. 2012;16:1011-9.

6. Mercer $\mathrm{CH}$, Copas AJ, Sonnenberg $\mathrm{P}$, Johnson AM, McManus S, Erens B, Cassell JA. Who has sex with whom? Characteristics of heterosexual partnerships reported in a national probability survey and implications for STI risk. Int J Epidemiol. 2009;38(1):206-14.

7. Erbelding EJ, Hummel B, Hogan T, Zenilman J. High rates of depressive symptoms in STD clinic patients. Sex Transm Dis. 2001;28(5):281-4.

8. Cook RL, Clark DB. Is there an association between alcohol consumption and sexually transmitted diseases? A systematic review. Sex Transm Dis. 2005;32(3):156-64

9. Martikainen P, Bartley M, Lahelma E. Psychosocial determinants of health in social epidemiology. Int J Epidemiol. 2002;31:1091-3.

10. Edelman NL, de Visser RO, Mercer $\mathrm{CH}$, McCabe L, Cassell JA. Targeting sexual health services in primary care: A systematic review of the psychosocial correlates of adverse sexual health outcomes reported in probability surveys of women of reproductive age. Prev Med. 2015;81:345-56.

11. Grobman WA, Stamilio DM. Methods of clinical prediction. Am J Obstet Gynecol. 2006;194(3):888-94.

12. WHO. Developing sexual health programmes: A framework for action. Geneva: World Health Organisation; 2010.

13. Mercer CH, Tanton C, Prah P, Erens B, Sonnenberg P, Clifton S, Macdowall W, Lewis R, Field N, Datta J, et al. Changes in sexual attitudes and lifestyles in Britain through the life course and over time: findings from the National Surveys of Sexual Attitudes and Lifestyles (Natsal). Lancet. 2013;382(9907):1781-94.

14. Erens B, Phelps A, Clifton S, Mercer CH, Tanton C, Hussey D, Sonnenberg P, Macdowall W, Field N, Datta J, et al. Methodology of the third British
National Survey of Sexual Attitudes and Lifestyles (Natsal-3). Sex Transm infect. 2014;90(2):84-9.

15. Mitchell KR, Mercer CH, Ploubidis GB, Jones KG, Datta J, Field N, Copas AJ, Tanton C, Erens B, Sonnenberg P, et al. Sexual function in Britain: findings from the third National Survey of Sexual Attitudes and Lifestyles (Natsal-3) Lancet. 2013;382(9907):1817-29.

16. Macdowall W, Gibson L, Tanton C, Mercer CH, Lewis R, Clifton S, Field N, Datta J, Mitchell KR, Sonnenberg P, et al. Lifetime prevalence, associated factors, and circumstances of non-volitional sex in women and men in Britain: findings from the third National Survey of Sexual Attitudes and Lifestyles (Natsal-3). Lancet. 2013;382(9907):1845-55.

17. Mercer $\mathrm{CH}$, Wellings $\mathrm{K}$, Johnson AM. What's new about Natsal-3? Sex Transm Infect. 2014:90(2):80-1.

18. Fenton KA, Mercer CH, Johnson AM, Byron CL, McManus S, Erens B, Copas AJ, Nanchahal K, Macdowall W, Wellings K. Reported Sexually Transmitted Disease Clinic Attendance and Sexually Transmitted Infections in Britain: Prevalence, Risk Factors, and Proportionate Population Burden. J Infect Dis. 2005;191(S1):S127-38.

19. Grulich AE, de Visser RO, Badcock PB, Smith AMA, Richters J, Rissel C, Simpson JM. Knowledge about and experience of sexually transmissible infections in a representative sample of adults: the Second Australian Study of Health and Relationships. Sex Health. 2014;11(5):481-94.

20. Sonnenberg P, Clifton S, Beddows S, Field N, Soldan K, Tanton C, Mercer CH, da Silva FC, Alexander S, Copas AJ, et al. Prevalence, risk factors, and uptake of interventions for sexually transmitted infections in Britain: findings from the National Surveys of Sexual Attitudes and Lifestyles (Natsal). Lancet. 2013;382(9907):1795-806.

21. Santelli JS, DiClemente RJ, Miller KS, Kirby D. Sexually transmitted diseases, unintended pregnancy, and adolescent health promotion. Adolesc Med 1999:10(1):87-108.

22. Steel $J L$, Herlitz CA. The association between childhood and adolescent sexual abuse and proxies for sexual risk behavior: A random sample of the general population of Sweden. Child Abuse Negl. 2005:29(10):1141-53.

23. Wellings K, Jones KG, Mercer CH, Tanton C, Clifton S, Datta J, Copas AJ, Erens B, Gibson LJ, Macdowall W, et al. The prevalence of unplanned pregnancy and associated factors in Britain: Findings from the third National Survey of Sexual Attitudes and Lifestyles (Natsal-3). Lancet. 2013;382(9907):1807-16

24. Wellings K, Nanchahal K, Macdowall W, McManus S, Erens B, Mercer CH, Johnson AM, Copas AJ, Korovessis C, Fenton KA, et al. Sexual behaviour in Britain: early heterosexual experience. Lancet. 2001;358(9296):1843-50.

25. Low N, Daker-White G, Barlow D, Pozniak AL. Gonorrhoea in inner London: results of a cross sectional study. Br Med J. 1997;314:1719-24.

26. Malarcher S. In: Malarcher S, editor. Social determinants of sexual and reproductive health: Informing future research and programme implementation. Geneva: World Health Organization; 2010.

27. Bland JM, Altman DG. Multiple significance tests: the Bonferroni method. BMJ 1995:310(6973):170

28. Tyler CP, Whiteman MK, Kraft JM, Zapata LB, Hillis SD, Curtis KM, Anderson J, Pazol K, Marchbanks PA. Dual Use of Condoms With Other Contraceptive Methods Among Adolescents and Young Women in the United States. J Adolesc Health. 2014:54(2):169-75.

29. Xaverius PK, Tenkku LE, Salas J. Differences Between Women at Higher and Lower Risk for an Unintended Pregnancy. Womens Health Issues. 2009;19(5):306-12.

30. Tornello SL, Riskind RG, Patterson CJ. Sexual Orientation and Sexual and Reproductive Health Among Adolescent Young Women in the United States. J Adolesc Health. 2014;54(2):160-8.

31. Everett BG. Sexual orientation disparities in sexually transmitted infections: examining the intersection between sexual identity and sexual behavior. Arch Sex Behav, 2013:42(2):225-36.

32. Victor EC, Chung R, Thompson Jr RJ. Identifying Adolescent Patients at Risk for Sexually Transmitted Infections: Development of a Brief Sexual Health Screening Survey. Clin Pediatr. 2015;54(9):878-87.

33. Gotz HM, van Bergen JE, Veldhuijzen IK, Broer J, Hoebe CJ, Steyerberg EW, Coenen AJ, de Groot F, Verhooren MJ, van Schaik DT, et al. A prediction rule for selective screening of Chlamydia trachomatis infection. Sex Transm Infect. 2005;81(1):24-30.

34. Haukoos JS, Lyons MS, Lindsell CJ, Hopkins E, Bender B, Rothman RE, Hsieh YH, Maclaren LA, Thrun MW, Sasson C, et al. Derivation and 
validation of the Denver Human Immunodeficiency Virus (HIV) risk score for targeted HIV screening. Am J Epidemiol. 2012;175(8):838-46.

35. Falasinnu T, Gilbert M, Gustafson P, Shoveller J. Deriving and validating a risk estimation tool for screening asymptomatic chlamydia and gonorrhea. Sex Transm Dis. 2014;41(12):706-12.

36. Bigrigg A, Nandwani R, llett R, Thow C, Lamont M, Bankowska U, Brechin S. Use of a staff administered structured questionnaire to identify relevant lifestyle issues and social-health determinants in a sexual and reproductive health service. Eur J Contracept Reprod Health Care. 2005;10(1):66-72.

37. Nathans LL, Oswald FL, Nimon K. Interpreting Multiple Linear Regression: A Guidebook of Variable Importance. Pract Assess Res Eval. 2012;17(9):1-19.

38. Mercer CH, Bailey JV, Johnson AM, Erens B, Wellings K, Fenton KA, Copas AJ. Women Who Report Having Sex With Women: British National Probability Data on Prevalence, Sexual Behaviors, and Health Outcomes. Am J Public Health. 2007:97(6):1126-33.

39. Marmot M, Wilkinson R, editors. Social Determinants of Health. 2nd ed. Oxford: Oxford University Press; 2006.

40. DH. A framework for sexual health improvement in England. London: Department of Health; 2013.

\section{Submit your next manuscript to BioMed Central} and we will help you at every step:

- We accept pre-submission inquiries

- Our selector tool helps you to find the most relevant journal

- We provide round the clock customer support

- Convenient online submission

- Thorough peer review

- Inclusion in PubMed and all major indexing services

- Maximum visibility for your research

Submit your manuscript at www.biomedcentral.com/submit 